\title{
Journal of Herbmed Pharmacology
}

Author's Reply

\section{Response to "Ipolamiide and other phytoconstituents of the Gynura procumbens"}

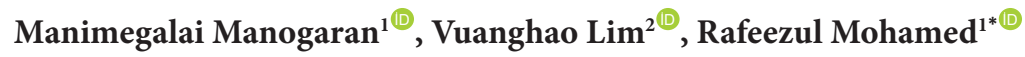 \\ ${ }^{1}$ Regenarative Medicine Cluster, Advanced Medical and Dental Institute, Universiti Sains, Malaysia \\ ${ }^{2}$ Integrative Medicine Cluster, Advanced Medical and Dental Institute, Universiti Sains, Malaysia
}

Received 17 October 2019, Accepted 20 November 2019

$\mathrm{W}$ e appreciate the constructive comments by Mohsen and Mozafar in our paper which appeared to have insufficient accuracy in analyzing the compounds present in Gynura procumbens in regards to their molecular weights. Regretfully, we admit our mistake as the $\mathrm{m} / \mathrm{z}$ stated in our published paper were mistakenly presented as unprocessed data, which were the $\mathrm{m} / \mathrm{z}$ with the adducts ion. Therefore, we have revised the Tables 1-5 with new calculated $\mathrm{m} / \mathrm{z}$ values as below: In addition, we also found that the compound of 2,3-dinor

Table 1. Compounds identified in Gynura procumbens ethanol extract (positive and negative ion modes) using LC-MS

\begin{tabular}{llll}
\hline Compound & Formula & $\mathrm{m} / \mathbf{z}$ & Group \\
\hline$\alpha-9(10)$-EpODE & $\mathrm{C}_{18} \mathrm{H}_{30} \mathrm{O}_{3}$ & 294.2120 & Fatty acid \\
$9 Z, 12 Z, 15 E-$ octadecatrienoic acid & $\mathrm{C}_{18} \mathrm{H}_{30} \mathrm{O}_{2}$ & 278.2169 & Fatty acid (Linolenic acid) \\
$6 \mathrm{E}, 9 \mathrm{E}-$ octadecadienoic acid & $\mathrm{C}_{18} \mathrm{H}_{32} \mathrm{O}_{2}$ & 280.2329 & Fatty Acyls \\
Pheophorbide a & $\mathrm{C}_{35} \mathrm{H}_{36} \mathrm{~N}_{4} \mathrm{O}_{5}$ & 592.2770 & Product of chlorophyll breakdown \\
\hline
\end{tabular}

Table 2. Compounds identified in the Gynura procumbens aqueous fraction (positive and negative ion modes) using LC-MS

\begin{tabular}{llc}
\hline Compound & Formula & m/z \\
\hline Isovitexin 2"-O-arabinoside & $\mathrm{C}_{26} \mathrm{H}_{28} \mathrm{O}_{14}$ & 564.1563 \\
Homoesperetin 7-rutinoside & $\mathrm{C}_{29} \mathrm{H}_{36} \mathrm{O}_{15}$ & 624.2401 \\
9Z,12Z,15E-octadecatrienoic acid & $\mathrm{C}_{18} \mathrm{H}_{30} \mathrm{O}_{2}$ & 278.2321 \\
Ipolamiide & $\mathrm{C}_{17} \mathrm{H}_{26} \mathrm{O}_{11}$ & 406.1822 \\
6,8-Di-C-beta-D-arabinopyranosylapigenin & $\mathrm{C}_{25} \mathrm{H}_{26} \mathrm{O}_{13}$ & Terpene glycoside glycoside \\
\hline
\end{tabular}

Table 3. Compounds identified in Gynura procumbens chloroform fraction (positive and negative ion modes) using LC-MS

\begin{tabular}{llll}
\hline Compound & Formula & $\mathrm{m} / \mathrm{z}$ & Group \\
\hline 4-(2-Hydroxypropoxy)-3,5-dimethylphenol & $\mathrm{C}_{11} \mathrm{H}_{16} \mathrm{O}_{3}$ & 196.1173 & Phenols \\
11-hydroperoxy-12,13-epoxy-9-octadecenoic acid & $\mathrm{C}_{18} \mathrm{H}_{32} \mathrm{O}_{5}$ & 328.2590 & Octadecanoids \\
Decenedioic acid & $\mathrm{C}_{10} \mathrm{H}_{16} \mathrm{O}_{4}$ & 200.1384 & Fatty Acyls \\
(-)-12-hydroxy-9,10-dihydrojasmonic acid & $\mathrm{C}_{12} \mathrm{H}_{20} \mathrm{O}_{4}$ & 228.1285 & Oxo monocarboxylic acid \\
5,8,12-trihydroxy-9-octadecenoic acid & $\mathrm{C}_{18} \mathrm{H}_{34} \mathrm{O}_{5}$ & 330.2329 & Fatty Acyls \\
(6S)-dehydrovomifoliol & $\mathrm{C}_{13} \mathrm{H}_{18} \mathrm{O}_{3}$ & 222.1327 & Sesquiterpenoids (terpenes) \\
\hline
\end{tabular}

*Corresponding author: Rafeezul Mohamed,

Email: rafeezul@usm.my 
Table 4. Compounds identified in Gynura procumbens ethyl acetate fraction (positive and negative ion modes) using LC-MS

\begin{tabular}{lllc}
\hline Compound & Formula & m/z & Group \\
\hline p-Salicylic acid & $\mathrm{C}_{7} \mathrm{H}_{6} \mathrm{O}_{3}$ & 138.0389 & Benzene \\
Luteolin 7-rhamnosyl(1->6)galactoside & $\mathrm{C}_{27} \mathrm{H}_{30} \mathrm{O}_{15}$ & 594.1664 & Flavonoid \\
6-Hydroxy luteolin 5-rhamnoside & $\mathrm{C}_{21} \mathrm{H}_{20} \mathrm{O}_{11}$ & 448.1084 & Flavonoid \\
Glycerol trivalerate & $\mathrm{C}_{18} \mathrm{H}_{32} \mathrm{O}_{6}$ & 344.2541 & Fatty acids \\
Formononetin 7-O-glucoside-6"-O-malonate & $\mathrm{C}_{25} \mathrm{H}_{24} \mathrm{O}_{12}$ & 516.1349 & Isoflavonoids \\
\hline
\end{tabular}

Table 5. Compounds identified in Gynura procumbens hexane fraction (positive and negative ion modes) using LC-MS

\begin{tabular}{lllll}
\hline Compound & Formula & m/z & Group \\
\hline Harderoporphyrin & $\mathrm{C}_{35} \mathrm{H}_{36} \mathrm{~N}_{4} \mathrm{O}_{6}$ & 608.2718 & Porphyrins \\
6E,9E-octadecadienoic acid & $\mathrm{C}_{18} \mathrm{H}_{32} \mathrm{O}_{2}$ & 280.2329 & Fatty Acyls \\
Pheophorbide a & $\mathrm{C}_{35} \mathrm{H}_{36} \mathrm{~N}_{4} \mathrm{O}_{5}$ & 592.2770 & Product of chlorophyll breakdown \\
9Z,12Z,15E-octadecatrienoic acid & $\mathrm{C}_{18} \mathrm{H}_{30} \mathrm{O}_{2}$ & 278.2321 & Fatty acid (Linolenic acid) \\
\hline
\end{tabular}

Thromboxane B1 was also wrongly mentioned as identified in G. procumbens ethyl acetate fraction (positive and negative ion modes) using LC-MS in our published paper (Table 4) (1). The correct compound is Glycerol trivalerate, $\mathrm{C}_{18} \mathrm{H}_{32} \mathrm{O}_{6}, \mathrm{~m} / \mathrm{z}$ value of 344.2541 from fatty acid group (Table 4) (2).

\section{Conflict of interests}

The authors declare that they have no conflict of interest.

\section{Ethical considerations}

Not applicable.

\section{References}

1. Manogaran M, Lim V, Mohamed R. Phytoconstituents of the Gynura procumbens ethanol leaf extract and its fractions and their effects on viability of macrophages. J Herbmed Pharmacol. 2019;8(3):224-30. doi: 10.15171/jhp.2019.33

2. Glycerol-trivalerate. Pubchem. Available from: https://pubi chem.ncbi.nlm.nih.gov//\#query=Glycerol-trivalerate. 